\title{
Qualitätsmanagement auch bei der Autoverwertung?
}

\author{
Prof. Dr.-Ing. habil. Gerd Stegemann, Dr.-Ing. Ingolf Wohlfahrt, Dipl.-Ing. Horst O. Friese
}

\section{Charakteristika der Autoverwertung}

Alle Welt spricht heute von Qualität, Qualitätsmanagement (QM) oder QM-Systemen. Begriffe, die sich auf den ersten Blick schwer mit Schrottauto, Autowrackplatz oder Shredderleichtmiillfraktion in Verbindung bringen lassen. Tatsächlich zeigen Erfahrungen der Autoren, daß es (heute noch) vielen Autorverwertungsunternehmen schwerfällt, sich ihre Tätigkeit eingebettet in den Rahmen eines systematisch gestalteten und zielgerichtet realisierten QM-Systems vorzustellen.

Ist damit die Titelfrage beantwortet? Sicher nicht, zumal davon auszugehen ist, daß sich die Recyclinglandschaft national und international, z. B. auf Grund eines geschärften Umweltbewußtseins, der Verknappung natuirlicher Ressourcen und des Deponierraumes, sich verändernden rechtlichen Rahmenbedingungen sowie sich wandelnden marktwirtschaftlichen Bedingungen, grundsätzlich verändern wird.

Ausgangspunkt für die Beantwortung der Frage, systematisches Qualitätsmanagement bei der Autoverwertung ja oder nein bzw. auf welcher Grundlage und in welchem Umfang, kann deshalb nur das unter diesen Bedingungen zu entwickelnde System der Autoverwertung sein, welches wiederum in ein Gesamtsystem Entwicklung, Fertigung und Nutzung von Autos einzupassen ist. Es ist nicht Aufgabe dieses Beitrages, ein derartiges System zu entwickeln. Vielmehr dienen nachfolgend aufgeführte Kennzeichen der Autoverwertung der Charakterisierung der Aufgabenstellung für das Qualitätsmanagement und der Begrenzung des Betrachtungsbereiches. Als typische Kennzeichen eines zukuinftigen Systems der Autoverwertung werden angesetzt:

- Abfallvermeidung mit dem Ziel einer drastischen Reduzierung zu deponierender Abfälle,

- weitgehende Realisierung der Kreislaufwirtschaft (Bauteil-/Baugruppen-/Stoffkreisläufe),

- vielfältige Verwertungsvarianten für Komponenten und Wertstoffe aus Altautos, z. B. durch originäre Wiederverwendung von Bauteilen und Baugruppen durch die Autoverwertungsunternehmen mit einem Trend zur rechnerunterstiitzten iiberregionalen Vermarktung sowie die industriemäßige stoffliche und energetische Verwertung von Komponenten und Wertstoffen, z. B. durch die Automobilindustrie bzw. ihre Systemlieferanten.

Wesentliches Glied in einem flächendeckenden Netz zur Erfassung, Riicknahme und Verwertung von Altautos ist der Autoverwertungsfachbetrieb, ein Klein- oder Mittelunternehmen. Auf ihn beziehen sich nachfolgende Ausfuih- rungen. Kennzeichen der Arbeit dieser Unternehmen sind: - Typenoffenheit und eine damit einhergehende große Produktvarianz,

- Übergang von Einzelarbeitsplätzen zur Losbildung gleichartiger Produkte und arbeitsteiligem Prozeßablauf auf der Basis von Fließbandkonzepten,

- Verwertung von iiberwiegend moralisch und physisch verschlissenen Autos mit einem Alter zwischen 8 und 15 Jahren (Abb. 1). Dazu kommen Autos jüngeren Alters, z. B. infolge von Unfällen, Diebstählen mit Beraubung oder Randale mit Beschädigungen,

- mögliche Verwertungsarten nach Abb. 2.

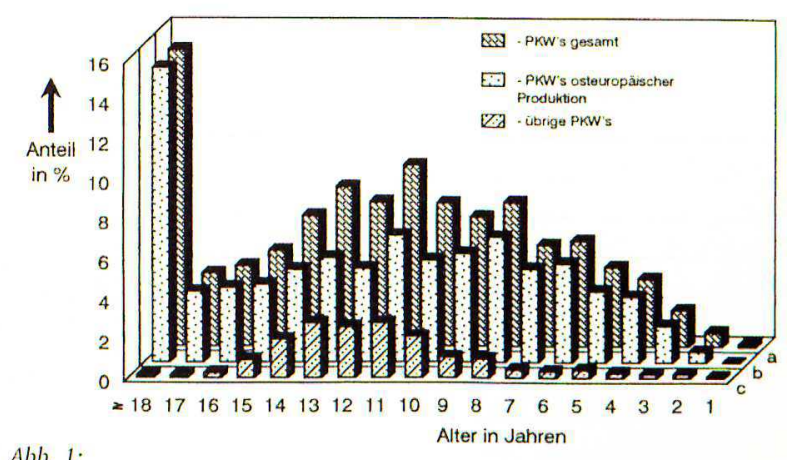

Altersstruktur der verwerteten PKW's eines Autoverwertungsfachbetriebes

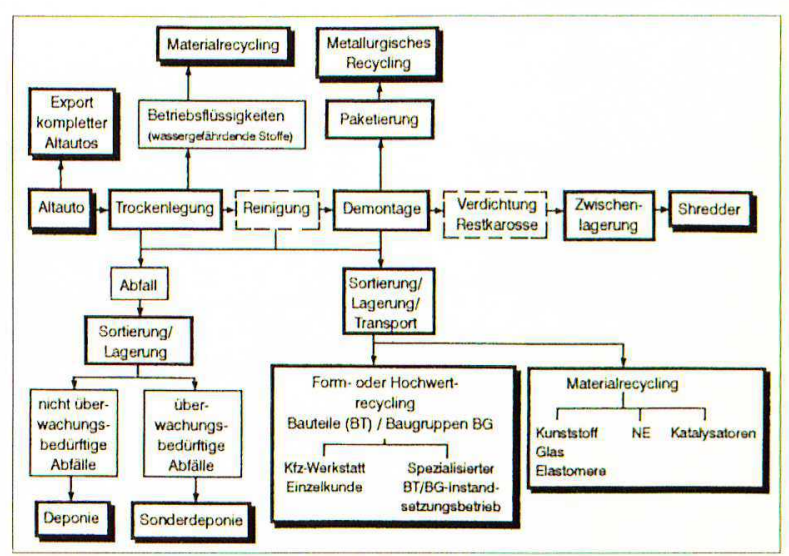

Abb. 2:

Mögliche Verwertungsarten für Altautos aus der Sicht des Autoverwerters

Es zeigt sich, daß neben traditionellen Aufgaben, wie Trockenlegung und Entnahme ausgewählter Bauteile/ -gruppen, in immer stärkerem Maße eine in wirtschaftlichen Grenzen realisierbare gezielte Demontage und Entfrachtung der Altautos erfolgt, außerdem im Rahmen der Verwertung Reinigungsaufgaben erforderlich sowie die Sortierung, Vorverdichtung bzw. die Vorzerkleinerung, die Lagerung und der Transport von Komponenten und Wertstoffen zu organisieren sind. 
Als wesentliches wirtschaftliches Standbein des Autoverwertungsfachbetriebes wird der Verkauf von Bauteilen/Baugruppen (originäre Wiederverwendung), gegebenenfalls nach Teilinstandsetzung und in jedem Fall nach Qualitätspruifung, angesehen. Bei einem Preis von 10 bis $40 \%$ des Neuteiles gewährt er eine Händlergarantie. Branchentypische Kennzeichungen, z. B. mit Gütezeichen, „Blauer Engel“" u. ä., scheinen zur Verbesserung der Kundenakzeptanz möglich. Das Kundenspektrum des Autoverwertungsfachbetriebes umfaßt:

- Recycling-Nerarbeitungs-/Shredderunternehmen,

- Kunden im Ersatzteilgeschäft, z. B. Kfz-Werkstätten,

- Bauteil-/Baugruppeninstandsetzungsbetriebe,

- Automobilindustrie.

Die sich daraus andeutende Forderungsvielfalt und der teilweise "fertigungstechnische" Charakter der Autorverwertung zwingen zur Systematisierung des Qualitätsmanagements im Rahmen der Autoverwertung.

\section{Grundlagen für die Gestalltung von QM-Systemen}

Qualität im allgemeinsten Sinne ist die Erfuillung von Kundenforderungen. Es ist davon auszugehen, daß die Beschaffenheit des vom Letztbesitzer angebotenen Altautos bezuiglich seiner Elemente sehr inhomogen ist, determinierte Qualitätsforderungen sind ihm gegenüber nicht einforderbar (fast jedes Altauto wird abgenommen) (Abb. 3). Durch die Gestaltung des Vewertungsprozesses hat der Autoverwerter zu gewährleisten, daß die in Abhängigkeit von der Verwertungsart determinierte Qualitätsforderung fuir die Komponenten und Wertstoffe (im weiteren Angebotsprodukte) gegenüber dem Kunden erfuillt wird. Im Sinne dieses Artikels steht Verwertungsprozeß fuir den durch den Autoverwertungsfachbetrieb zu rea- lisierenden Dienstleistungsprozeß (Letztbesitzer Altauto/Kunde fuir Angebotsprodukte der Autoverwertung) und den eigentlichen „Fertigungsprozeß“ der Autoverwertung - den Recyclingprozeß. Die Prozeßgestaltung wird wie kaum in einer anderen Branche durch die berechtigten Interessen der Allgemeinheit mitgeprägt (Abb. 4).

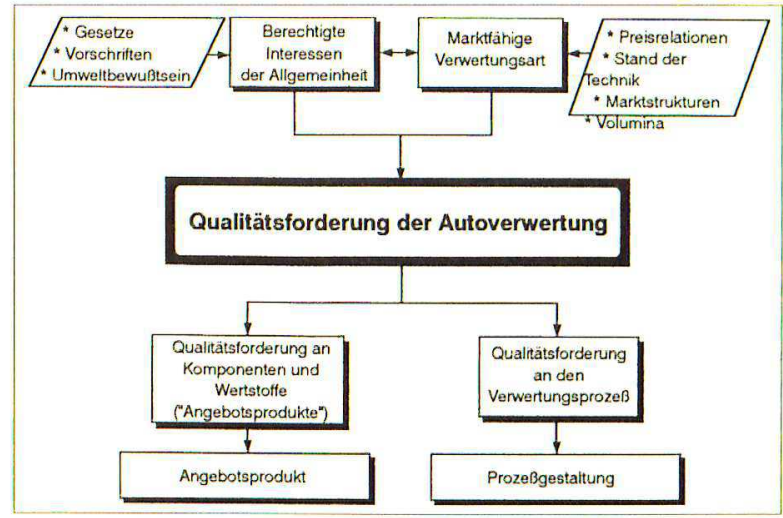

Abb. 4: Quellen und Ziele von Qualitätsforderungen der Autoverwertung

Die Ableitung der Qualitätsforderung fuir die Angebotsprodukte der Autoverwertung ist deshalb als erste und wichtige Aufgabe das Qualitätsmanagement anzusehen. Dabei ist zu berïcksichtigen, daß der Rang der Qualitätsforderung an das Angebotsprodukt stark durch die Verwertungsart bestimmt wird (Abb. 5).Während $\mathrm{z}$. B. für das metallurgische Recycling neben der Trockenlegung eine gezielte Entfrachtung des Objektes erfolgen muß, sind bei der originären Wiederverwendung von Bauteilen (Formrecycling) Forderungen zur Funktionsfähigkeit, Sicherheit und Zuverlässigkeit zu erfüllen. Den prinzipiellen Ablauf der Beschreibung der Qualitätsforderung durch Qualitätsmerkmale und für die Ableitung von Prüifmerkmalen zeigt Abb. 6.

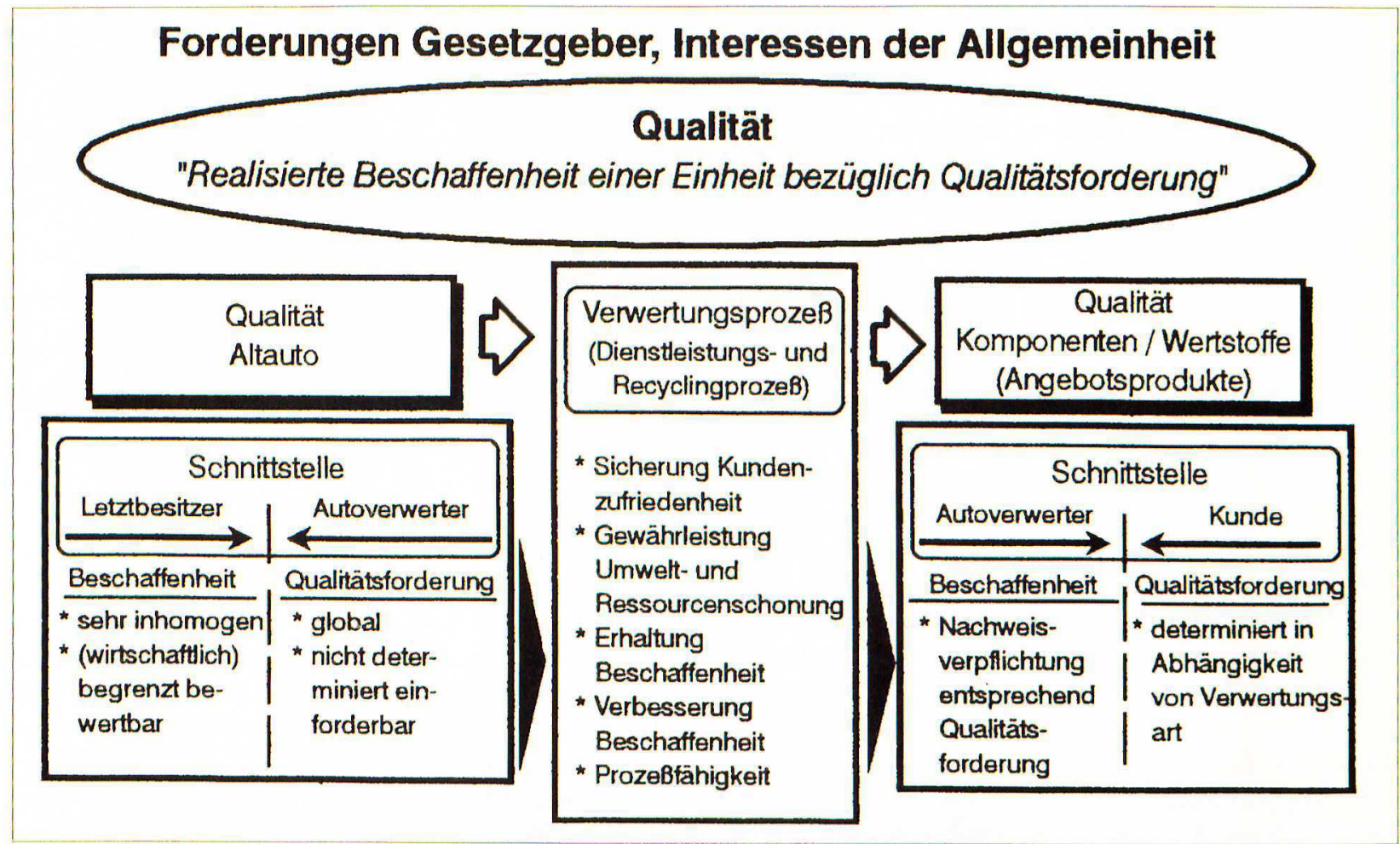




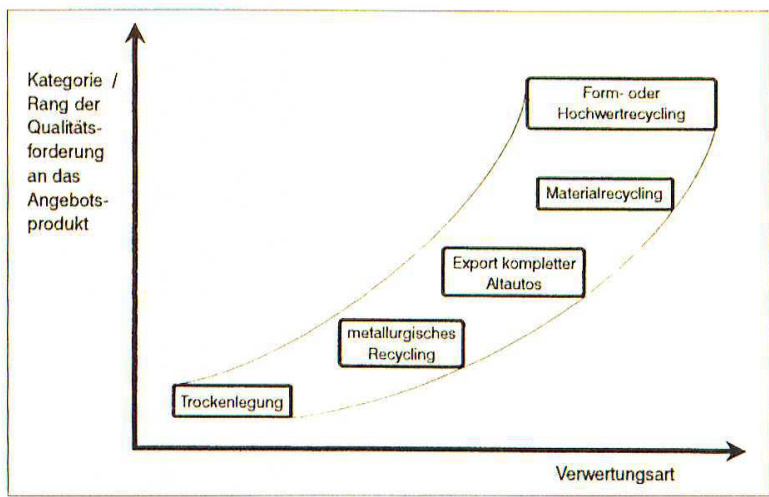

Abb. 5: Zusammenhang zwischen der Verwertungsart und der Kategorie/den Rang der Qualitätsforderung an Komponenten bzw. Wertstoffe (Angebots produkte) aus Sicht des Autoverwerters

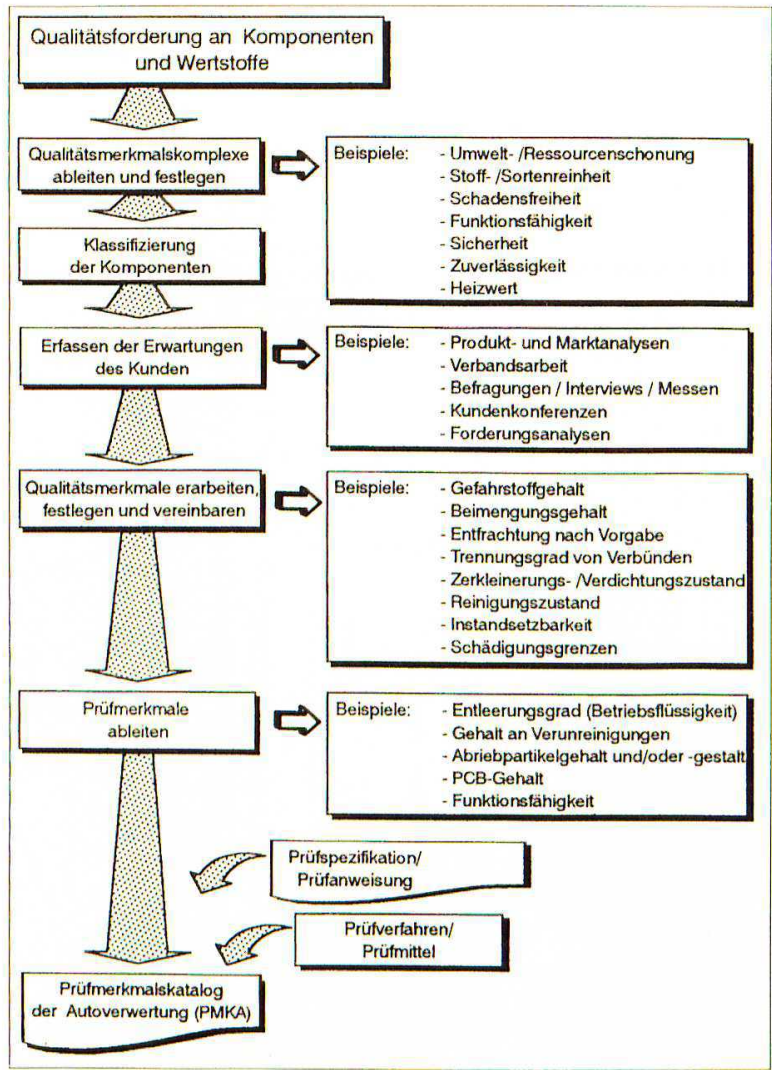

Abb. 6: Ableitung der Qualitäts- und Prïfmerkmale für Komponenten und Wertstoffe (Angebots-produkte) aus der Autoverwertung

Entsprechend der allgemeinen Erkenntnis, daß ein Produkt nicht besser sein kann als der zu seiner Herstellung genutzte Prozeß, kommt auch in der Autoverwertung der Prozeßgestaltung eine besondere Bedeutung zu. Die Abb. 7 vermittelt ausgewählte Qualitätsforderungen für die Gestaltung des Recyclingprozesses. Diese Darstellung unterstreicht die notwendige Verbindung von Qualitäts- und Umweltmanagement bei der Autoverwertung. Entsprechend der Untergliederung des Verwertungsprozesses im Dienstleistungs- und Recyclingprozeß wird empfohlen, die Gestaltung normenkonformer QM-Systeme für die Autorverwertung auf der Basis der Regelwerke DIN EN ISO 9004-1 und DIN ISO 9004-2 vorzunehmen (Abb. 8).

Erste Analysen zur Normenkonformität (DIN EN ISO 9002) qualitätsrelevanter Tätigkeiten bei der Autoverwertung verdeutlichen besonders große Defizite bezuig- lich der Dokumentation und Nachweisfithrung, insbesondere für den Recyclingsprozeß. Besondere Schwerpunkte fuir die Verfahrensforschung zum Recyclingprozeß ergeben sich aus den Abweichungen innerhalb des Prozeßelementes „Komponenten- und WertstoffIdentifikation" (s. Abb. 7), insbesondere begründet in unbefriedigenden Verfahren zur „Produktverifizierung“ und fehlenden Detektionsverfahren.

\section{Einrichtung von QM-Systemen}

Als wesentliche Gründe für die Einrichtung und Erhaltung von QM-Systemen bei der Autoverwertung werden - die Herstellung und Erhaltung der Qualitätsfähigkeit des Autoverwertungsfachbetriebes sowie

- der Nachweis der Qualitätsfähigkeit durch den Autoverwertungsfachbetrieb gegenüber seinem Kunden und gegenuiber der Allgemeinheit gesehen (Abb. 9).

Vorstellbar ist, daß die Zulassung zum Betrieb eines Autoverwertungsunternehmens ( $z$. B. auf der Grundlage eines Planfeststellungsverfahrens) und/oder die Autorisierung eines Autoverwertungsfachbetriebes, z. B. durch einen Automobilhersteller, mit dem Nachweis der Qualitätsfähigkeit des Autoverwertungsfachbetriebes auf der Grundlage eines normenkonformen QM-Systems gekoppelt wird.

Die Optimierung betrieblicher Abläufe und in der Gesamtheit des Verwertungprozesses muß sich, neben herkömmlichen Zielkriterien, wie z. B. zur Effektivität oder zur Gewährleistung des Umweltschutzes im Verwertungsprozeß, in stärkerem Maße an der Abfallvermeidung orientieren.

QM-Systeme bilden dann eine wesentliche Voraussetzung für Marktzugang und Marktanteil. Sie sind ein wichtiges Instrument zur Risikovermeidung/-begrenzung und der Ertragssicherung.

Abgeleitet aus der Tatsache, daß der Recyclingprozeß im Sinne dieses Artikels nicht schlechthin die Umkehrung der Arbeitsfolge der Montage in der Neufertigung ist, ergeben sich eine Reihe Spezifika für das Qualitätsmanagement der Autoverwertung, die letztlich branchenspezifische Lösungen für die Einrichtung und Erhaltung von QM-Systemen bei der Autoverwertung erfordern.

Wesentliche Schwerpunktaufgaben der Integration des Qualitätsmanagements in den Verwertungsprozeß von Altautos sind in Abb. 10 zusammengefaßt.

Es sei nochmals darauf hingewiesen, daß als Ausgangspunkt der Arbeiten zum Qualitätsmanagement in der Autoverwertung die Beschreibung der Qualitätsforderung unter Berücksichtigung wirtschaftlicher Realisierungsmöglichkeiten angesehen wird (s. Abb. 10: Q-Planung).

Die Zusammenfassung aller Aktivitäten zur Einrichtung und Erhaltung von QM-Systemen ist inhaltlich und terminlich genau zu planen, die Aufgaben in diesem Prozeß sind abzustecken und zu beschreiben.

Das in Abb. 11 dargestellte Ablaufschema läßt sich in drei Hauptabschnitte gliedem:

- Entscheidung zum „Warum QM“ durch die Unterneh- 


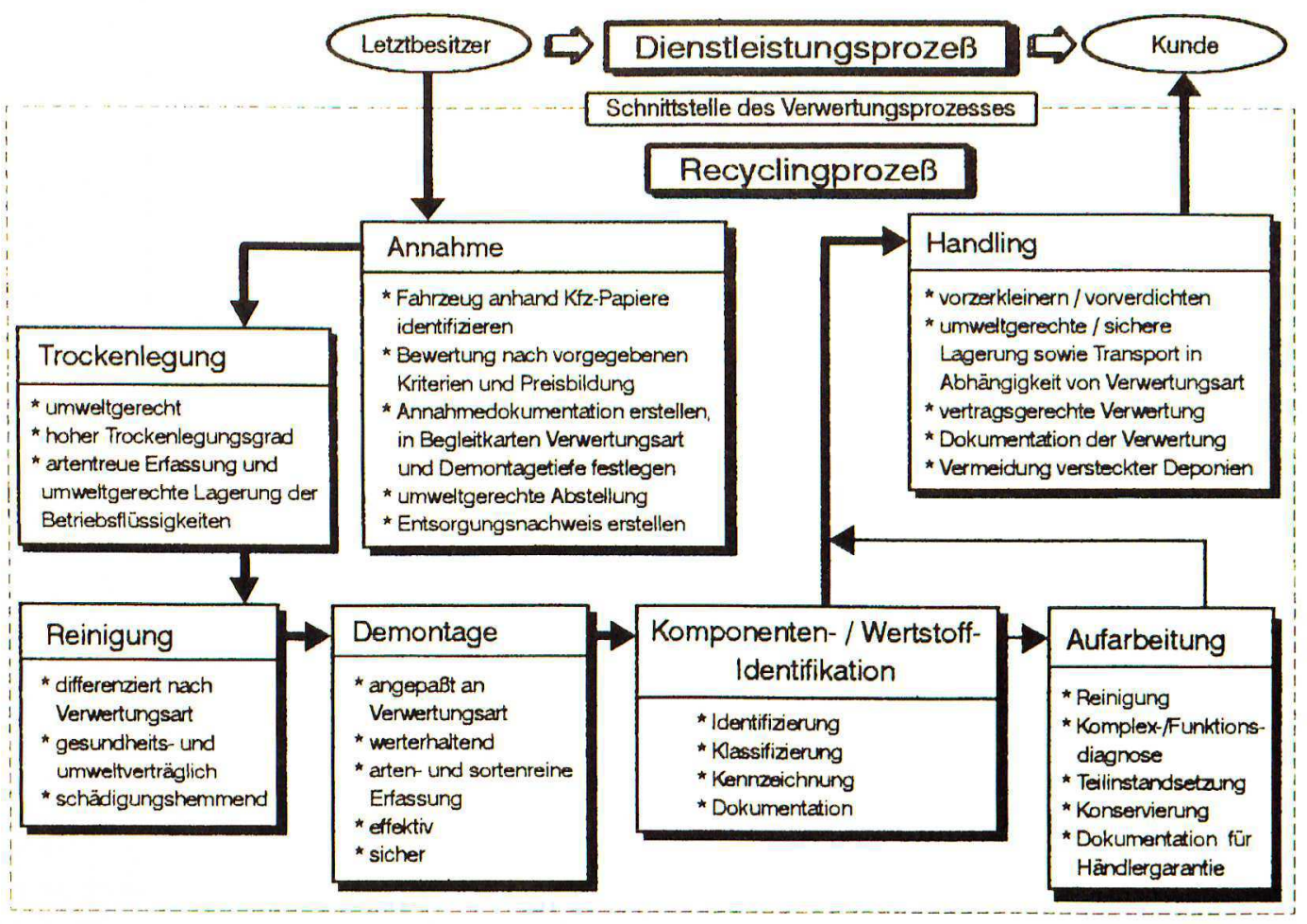

Abb. 7: Ausgewählte Qualitätsforderungen für die Gestlatung des Recycling-prozesses der Autoverwertung (nach Prozeßelementen)

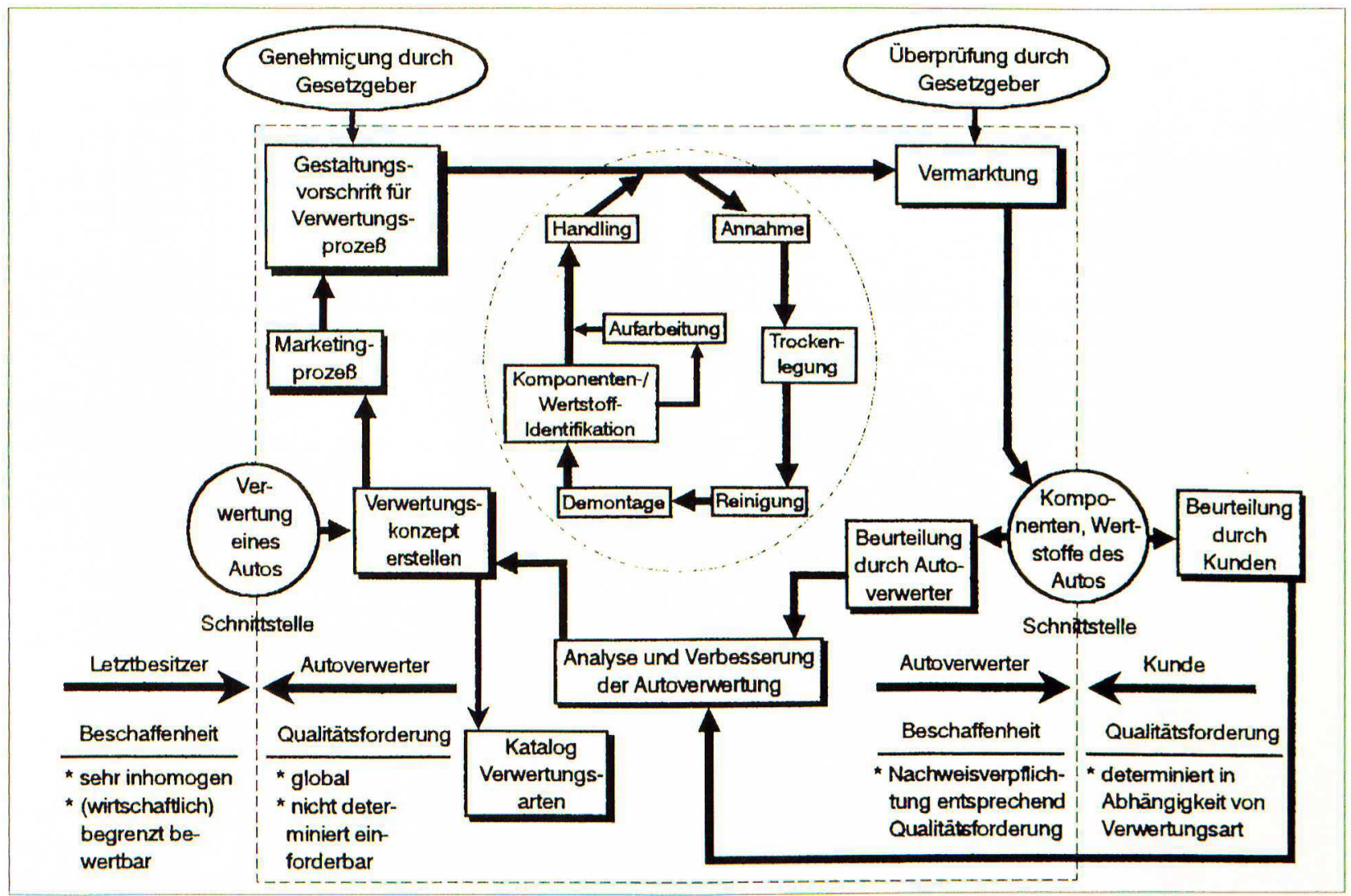

Abb. 8: „Äußserer“ (DIN ISO 9004-2) und ,innerer“ (DIN EN ISO 9004-1) „Qualitätskreis“ der Autoverwertung

mensfuihrung herbeifuihren (Verantworung der obersten Leitung). Aufwendungen für und Ziele von QMSystemen sind gründlich zu erarbeiten.

- Analyse des Systems qualitätsrelevanter Tätigkeiten im Unternehmen, Programm zur Erreichung der Qualitätsfähigkeit erarbeiten und realisieren (s. Abb. 11).

- Laufende Aktualisierung des QM-Systems.
Beispielhaft in Abb. 11 eingezeichnete Schnittstellen beinhalten folgende Arbeitsaufgaben:

1 Ziele/Aufgaben fuir normenkonforme QM-Systeme in Geschäftsführung erarbeiten,

2 Qualitätsphilosophie im Management beraten und Festlegungen treffen,

3 Mitarbeiterinformation und -einbeziehung, begin- 


\section{Gründe für Qualitätsmanagementsysteme}
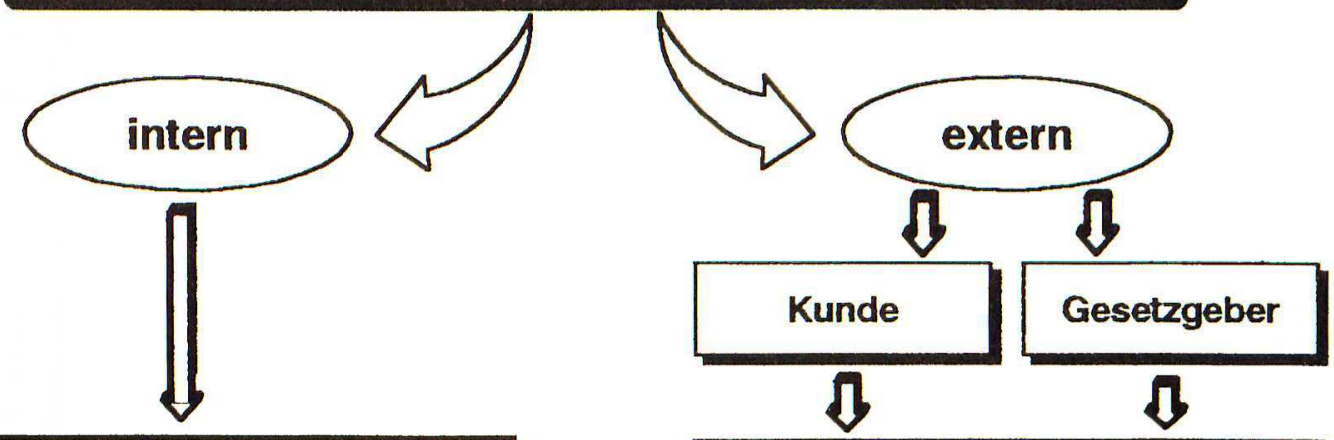

Herstellung und Erhaltung der

Qualitätsfähigkeit zur Gewährleistung:

* Unternehmenszulassung (Gesetz- / Auftraggeber)

* Optimierung betrieblicher Abläufe und des Verwertungsprozesses

* Marktanteil

* Ertragsverbesserung

Nachweis der Qualitätsfähigkeit zur Sicherung:

* Gewährleistung Q-Forderung Recyclat

* Marktzugang

* Gesetzes- und Normenkonformität

* Kundenzufriedenheit

* Risikovermeidung / -begrenzung

Abb. 9: Gründe für die Einrichtung und Erhaltung von Qualitätsmanage-mentsystemen bei der Autoverwertung

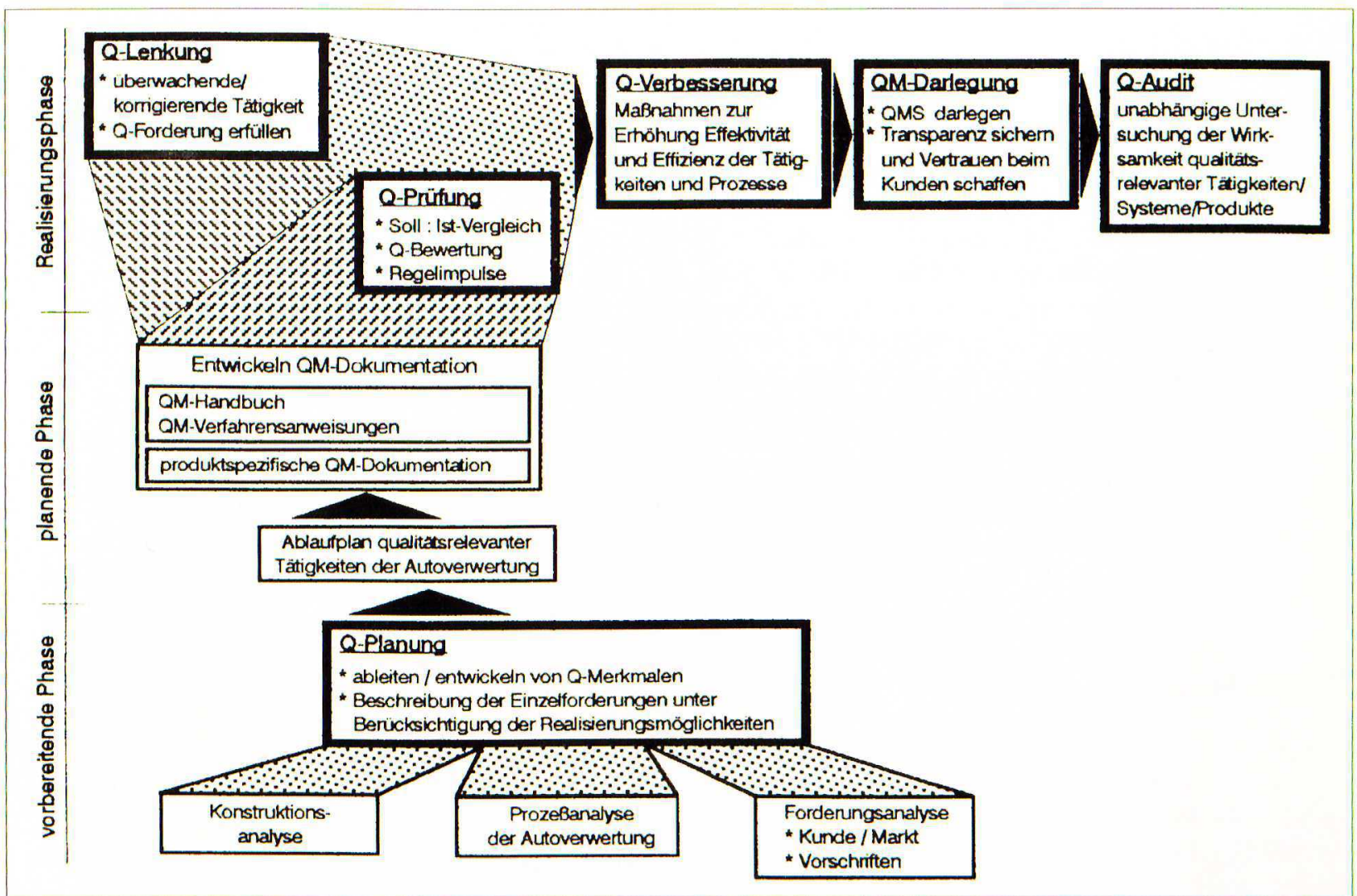

Abb. 10: Schwerpunktaufgaben bei der Integration des Qualitätsmanagements in den Vewertungsprozeß von Altautos

nend mit der Diskussion der Unternehmensgrundsätze zur Qualitätsarbeit,

4 Festlegung der Organisationseinheit, für die (zunächst) das QM-System erarbeitet werden soll,

5 Projektteam benennen und mit Verantwortung und Kompetenzen ausstatten,

6 umfassende Mitarbeiterschulung einleiten,
7 Grobkonzept für den Stufenplan zur Einrichtung eines normenkonformen QM-Systems erstellen,

8 Festlegung der Qualitätsaspekte in der Aufbau- und Ablauforganisation, Ergänzung der Stellenbeschreibungen für das Management um Aspekte der Qualitätsarbeit,

9 Kunden in die konzeptionelle Arbeit einbeziehen, 


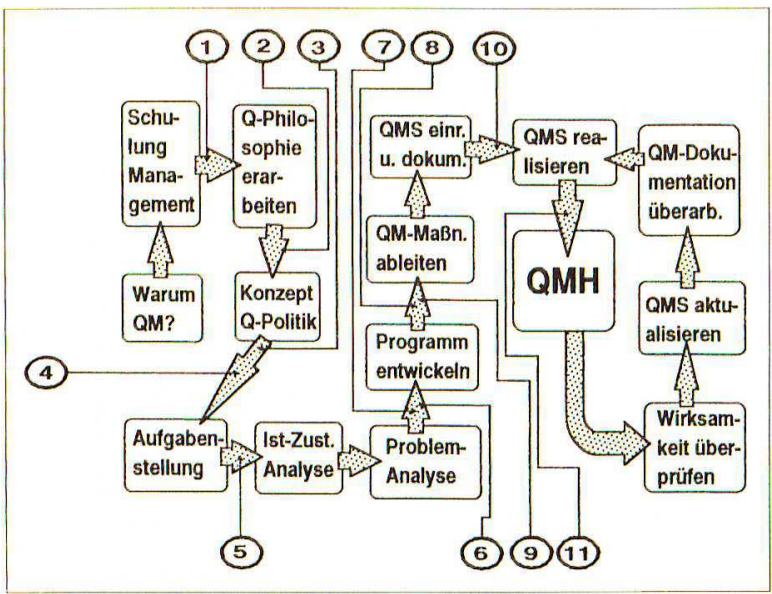

Abb. 11: Ablaufschema zur Erlangung der Qualitätsfähigkeit

10 Einheit von Erarbeitung und Überführung der QMDokumente sichern,

11 regelmäßige Bewertung der Wirksamkeit des QMSystems (interne Audits, Qualitätsreviews) durchfuihren und in der Geschäftsfuihrung bewerten.

\section{Zusammenfassung}

Im Artikel werden Charakteristika der Autoverwertung als Grundlage fuir die Ableitung von Aufgabenstellungen des Qualitätsmanagements in Autoverwertungsfachbetrieben beschrieben. Die Ergebnisse unterstreichen die Notwendigkeit der Einrichtung von QM-Systemen in der Autoverwertung bei Berïksichtigung der notwendigen Ganzheitlichkeit der Qualitätsstrategie in den Phasen Planung, Realisierung und Nutzung von Autos.

Davon ausgehend werden Voraussetzungen fuir die Gestaltung von QM-Systemen zusammengestellt und Ansatzpunkte fuir die Einrichtung von QM-Systemen in Autoverwertungsfachbetrieben erarbeitet. Als Basis fuir diese Arbeiten wird das Regelwerk DIN EN ISO 9004-1 und DIN ISO 9004-2 vorgeschlagen.

Die Darstellung des methodischen Apparates erfolgt am Beispiel der Autoverwertung, eine Übertragung der verallgemeinerungsfähigen Aussagen auf andere Produkte ist möglich.

\section{Verfasser}

Prof. Dr--Ing. habil. Gerd Stegemann

Technische Fachhochschule Wildau

Fachbereich Maschinenbau

Tel. (03375) 507-213

Dr.-lng. Ingolf Wohlfahrt

Technische Fachhochschule Wildau

Fachbereich Maschinenbau

Tel. (03375) 507-213

Dipl.-Ing. Horst O. Friese

Geschäftsführer, Autoverwertungscenter Neuenhagen 